The International Journal of Multimedia \& Its Applications (IJMA) Vol.3, No.1, February 2011

\title{
CONTOURLET TRANSFORM BASED WATERMARKING FOR COLOUR IMAGES
}

\author{
Dinesh Kumar $^{1}$ and Vijay Kumar ${ }^{2}$ \\ ${ }^{1}$ Department of Computer Science \& Engineering, GJUS\&T, Hisar, Haryana \\ dinesh_chutani@yahoo.com \\ ${ }^{2}$ Department of Computer Science \& Engineering, JCDMCOE, Sirsa, Haryana \\ vijaykumarchahar@gmail.com
}

\begin{abstract}
This paper presents a contourlet transform based watermarking scheme for colour images. It also makes use of discrete cosine transform and singular value decomposition. Discrete cosine transform is applied on three colour components $(Y, \mathrm{Cb}$ and $\mathrm{Cr})$, obtained from the colour image, to produce DCT coefficients. These coefficients are decomposed into directional subbands using 2- level contourlet transform. The watermark is also decomposed using contourlet transform. The fourth directional subbands of cover image and watermark as well are further subjected to Laplacian pyramid decomposition. As a result, the low pass subbands are obtained. The singular values of lowpass subband of watermark are embedded into singular values of lowpass subband of cover image. The experimental results reveal that the proposed watermarking scheme is robust against common image processing attacks. The results also demonstrate good imperceptibility.
\end{abstract}

\section{KEYWORDS}

Contourlet Transform (CT), Discrete Cosine Transform (DCT), Singular Value Decomposition (SVD)

\section{INTRODUCTION}

The technological advancement in the field of multimedia and the availability of high speed networks have enabled us to obtain, reproduce and distribute data with ease and with almost no loss in quality. Under such a scenario, the protection of digital data has become increasingly important. Digital watermarking is one among a number of solutions that can help provide copyright protection. It is being frequently used in applications such as medical imaging, digital camera and video-on-demand systems etc. [1].

The watermarking technique incorporates embedding of watermark into digital data that can later be extracted in order to claim the ownership of data. Such techniques are broadly categorized into two groups, the spatial domain and frequency domain watermarking techniques $[2,4,5]$. The former embeds watermarks in spatial characteristics of cover image directly whereas the latter transforms the cover image into frequency domain coefficients before embedding watermarks in it. The commonly used transforms in watermarking techniques are Discrete Wavelet Transform (DWT), Discrete Fourier Transform (DFT), Discrete Cosine Transform (DCT), and Singular Value Decomposition (SVD). DWT and SVD are more popular as compared to others. But these techniques do not possess the directional information such as directional edges of the image. Discrete Contourlet Transform (CT) is capable of capturing the directional information with multiresolution representation $[6,11]$. It makes use of Laplacian Pyramid for multiresolution representation of the image $[9,10]$ followed by a directional decomposition on every band pass image using directional filters $[8,10]$.

This paper explores a colour image watermarking scheme wherein Contourlet Transform has been used along with DCT and SVD. The remainder of this paper is organized as follows. Section 2 briefs about SVD, DCT and CT. The next section 3 covers the proposed colour image

DOI : 10.5121/ijma.2011.3111 
The International Journal of Multimedia \& Its Applications (IJMA) Vol.3, No.1, February 2011

watermarking scheme. Section 4 demonstrates the experimental results followed by conclusions in section 5 .

\section{BACKGROUND}

The following contains a brief description of Singular Value Decomposition, Discrete Cosine Transform and Contourlet Transform.

\subsection{Singular Value Decomposition}

The Singular Value Decomposition (SVD) technique has been widely used in large number of applications such as pattern recognition, image compression and watermarking $[2,15,16]$. Suppose we have an image represented by matrix $\mathbf{X}$ of size K rows and M columns; applying SVD on $\mathbf{X}$ will produce three matrices and $\mathbf{X}$ can be written as

$$
\mathbf{X}=\mathbf{U S V}^{\mathbf{T}}
$$

where matrices $\mathbf{U}$ and $\mathbf{V}$ are $K \times K$ and $M \times M$ orthogonal matrices respectively. $\mathbf{S}$ is a $K \times M$ diagonal matrix with singular values (SV) on the diagonal. The columns of $\mathbf{U}$ and $\mathbf{V}$ are the eigenvectors of $\mathbf{X} \cdot \mathbf{X}^{\mathbf{T}}$ and $\mathbf{X}^{\mathbf{T}} \cdot \mathbf{X}$ respectively. The Singular Values (SVs) in S matrix are the square roots of the eigenvalues of $\mathbf{X} \cdot \mathbf{X}^{\mathbf{T}}$ or $\mathbf{X}^{\mathbf{T}} \cdot \mathbf{X}$ [17]. The main property of SVD in respect of watermarking is that larger singular values of an image do not change significantly when image processing attacks are performed on the image [2].

\subsection{Discrete Cosine Transform}

The Discrete Cosine Transform (DCT) is a technique for converting a signal into elementary frequency components [2,3]. It is applied on an image $\mathbf{I}$, having $K \times M$ pixels to transform the image according to equation 2 [2]:

$$
y(u, v)=\sqrt{\frac{2}{M}} \sqrt{\frac{2}{K}} \alpha_{u} \alpha_{v} \sum_{m=0}^{M-1} \sum_{k=0}^{K-1} I(m, k) \cos \frac{(2 m+1) u \pi}{2 M} \cos \frac{(2 k+1) v \pi}{2 k}
$$

The values $y(u, v)$ are called the DCT coefficients of the image matrix. The values of $\alpha_{u}$ and $\alpha_{v}$ are both set to $1 / \sqrt{2}$ when $u, v=0$, otherwise 1 . The image can be reconstructed by applying IDCT according to equation 3 [2]:

$$
I(m, k)=\sqrt{\frac{2}{M}} \sqrt{\frac{2}{K}} \sum_{u=0}^{M-1} \sum_{v=0}^{K-1} \alpha_{u} \alpha_{v} y(u, v) \cos \frac{(2 m+1) u \pi}{2 M} \cos \frac{(2 k+1) v \pi}{2 k}
$$

\subsection{Contourlet Transform}

The Contourlet Transform (CT) was proposed by Do and Vetterlin $[6,11]$. The main feature of this transform is its efficiency in capturing two dimensional singularities like edges whereas wavelet transform capture only point singularities [7, 12]. It provides a multiresolution and directional expansion of images using Pyramidal Directional Filter Bank (PDFB). The PDFB is constructed by two filter-bank stages, a Lapalacian Pyramid (LP) followed by a Directional Filter Bank (DFB) [12]. The former captures point discontinuities and latter links these into liner structures. The LP decomposes the image into frequency band to obtain singular points. The DFB decomposes each LP detail band to capture directionality. 
The International Journal of Multimedia \& Its Applications (IJMA) Vol.3, No.1, February 2011

\section{Proposed Watermarking Scheme}

The scheme has embedding and extraction procedures. The steps for embedding and extraction are as follows:

\subsection{Embedding Procedure}

1. Apply 2-level CT on the watermark to obtain directional sub bands.

2. Apply LP decomposition on fourth directional subband to obtain bandpass image $\mathbf{W}$.

3. Perform SVD on bandpass image obtained from Step2.

$$
\mathbf{W}=\mathbf{U}_{\mathbf{w}} \times \mathbf{S}_{\mathbf{w}} \times \mathbf{V}_{\mathbf{w}}^{\mathbf{T}}
$$

where $\mathbf{W}$ is the bandpass image of watermark.

4. Transform the cover image from RGB colour space into YcbCr colour space.

5. For each colour component

(a) Perform DCT.

(b) Perform 2-level CT to obtained directional sub bands.

(c) Perform LP decomposition on fourth directional subband.

(d) Perform SVD on bandpass image obtained from Step 5-(c).

$$
\mathbf{C B P}_{\mathbf{i}}=\mathbf{U B P}_{\mathbf{i}} \times \mathbf{S B P}_{\mathbf{i}} \times \mathrm{VBP}_{\mathbf{i}}^{\mathrm{T}}
$$

where $C B P_{i}$ represent the fourth direction subband of cover image.

(e) Modify the singular values of bandpass image.

$$
\mathbf{S B P}_{\mathbf{i}}^{\prime}=\mathbf{S B P}_{\mathbf{i}}+\boldsymbol{\alpha} \times \mathbf{S}_{\mathbf{w}}
$$

where $S B P_{i}^{\prime}$ is modified singular matrix, $S B P_{i}$ is singular matrix of directional subband of cover image, $S_{w}$ is singular matrix of watermark and $\alpha$ is the amplification factor.

(f) Obtain the modified band pass image.

$$
\mathrm{CBP}_{\mathbf{i}}^{*}=\mathrm{UBP}_{\mathbf{i}} \times \mathrm{SBP}_{\mathbf{i}}^{\prime} \times \mathrm{VBP}_{\mathrm{i}}^{\mathrm{T}}
$$

where $C B P_{i}^{*}$ is modified bandpass of cover image, $U B P_{i}$ and $V B P_{i}$ are orthogonal matrices of bandpass of cover image.

(g) Apply LP reconstruction on modified bandpass image.

(h) Perform inverse CT (ICT).

(i) Perform IDCT.

6. Transform the image into RGB colour space to get the watermarked image.

\subsection{Extraction Procedure}

1. Transform the cover image and watermarked image from RGB colour space into $\mathrm{YCbCr}$ Space.

2. For each colour component of cover and watermarked images

(a) Perform DCT.

(b) Perform 2-level CT to obtained directional subbands.

(c) Perform LP decomposition on fourth directional subband.

(d) Perform SVD on bandpass image of cover and that of watermarked image.

$$
\begin{aligned}
& \mathrm{CBW}_{\mathrm{wi}}=\mathrm{UBW}_{\mathrm{wi}} \times \mathrm{SBW}_{\mathrm{wi}} \times \mathrm{VBW}_{\mathrm{wi}}^{\mathrm{T}} \\
& \mathrm{CBP}_{\mathrm{i}}=\mathrm{UBP}_{\mathrm{i}} \times \mathrm{SBP}_{\mathrm{i}} \times \mathrm{VBP}_{\mathrm{i}}^{\mathrm{T}}
\end{aligned}
$$


The International Journal of Multimedia \& Its Applications (IJMA) Vol.3, No.1, February 2011

where $C B W_{w i}$ and $C B P_{i}$ are bandpass of watermarked and cover images respectively .

(e) Extract singular values of watermark as

$$
\mathbf{S}_{\mathrm{w}}^{\mathrm{ext}}=\frac{\mathbf{S B W}_{\mathrm{wi}}-\mathbf{S B P}_{\mathbf{i}}}{\alpha}
$$

where $S B W_{w i}$ and $S B P_{i}$ are singular matrices of watermarked and cover images respectively. $S_{w}^{e x t}$ is extracted singular matrix of watermark.

3. Perform SVD on all bandpass images of watermark

$$
\mathbf{W}^{e x t}=\mathbf{U}_{\mathbf{w}} \times \mathbf{S}_{w}^{e x t} \times \mathbf{V}_{\mathbf{w}}^{\mathbf{T}}
$$

where $W^{e x t}$ is extracted watermark image.

4. Apply LP reconstruction on results obtained from Step 3.

5. Perform ICT to get the watermarks.

\section{EXPERIMENTATION AND RESULTS}

\subsection{Experiment 1 and Results}

The experimentation was done using MATLAB platform. As many as eight different cover images were taken. These images are: Lena, Barbara, Goldhill, Redfort, Boat, Taj Mahal, Statue of Liberty and Nuclear Plant, each of size $256 \times 256$. One gray scale image named Springer $\log$ of size $90 \times 256$ was taken as watermark. The CT uses 'pkva' for both Pyramid filter and Directional filter to obtain a two level decomposition. Amplification factor has been set to 0.01 for embedding watermark. The Springer logo is embedded into all the cover images. Figure 1 shows watermarked images and extracted watermark image using proposed method.

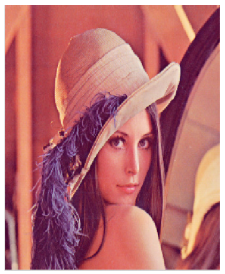

(a)

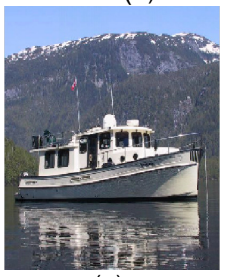

(e)

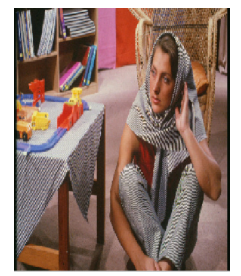

(b)

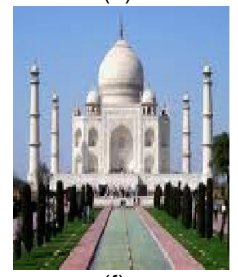

(f)

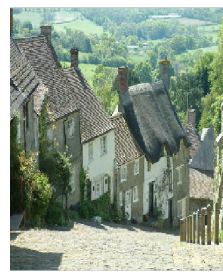

(c)

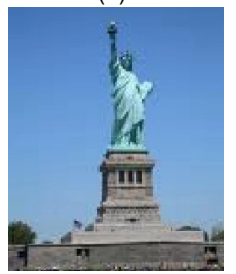

(g)

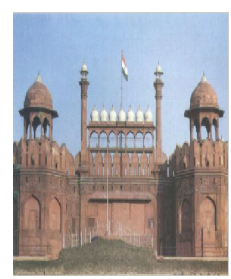

(d)

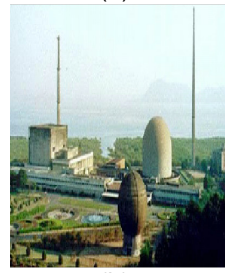

(h)

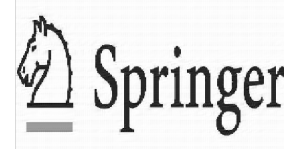

(i)

Figure 1. Watermarked images; (a) Lena (b) Barbara (c) Goldhill (d) Redfort (e) Boat (f) Taj (g) Statue (h) Nuclear and Extracted watermark image; (i) Springer Logo

The Peak Signal to Noise Ratio (PSNR), Structural Similarity Index (SSIM) [13] and 
The International Journal of Multimedia \& Its Applications (IJMA) Vol.3, No.1, February 2011

correlation coefficient values of each watermarked image are tabulated in table 1.

Table 1. Quality metrics of watermarked images and correlation coefficient using Proposed Method.

\begin{tabular}{|l|l|l|l|l|l|l|l|l|}
\hline & Lena & Barbara & Goldhill & Redfort & Boat & Taj & Status & Nuclear \\
\hline PSNR & 43.2226 & 42.6960 & 43.1691 & 43.1228 & 43.0486 & 43.4269 & 42.1642 & 43.7958 \\
\hline SSIM & 0.9845 & 0.9947 & 0.9976 & 0.9875 & 0.9915 & 0.9856 & 0.9682 & 0.9815 \\
\hline $\begin{array}{l}\text { Cor. } \\
\text { Coef. }\end{array}$ & 0.9940 & 0.9941 & 0.9936 & 0.9932 & 0.9930 & 0.9966 & 0.9962 & 0.9942 \\
\hline
\end{tabular}

Table 1 shows that the proposed method yields much better results so far as the quality of images is concerned. We also obtain better correlation coefficient using the proposed scheme.

\subsection{Experiment 2 and Results}

The next experiment was performed to see the effect of different image processing attacks such as Sharpening, Gamma Correction, Affine Transform, Histogram Equalization, Resizing, Cropping, Rotation and Gaussian blur. Figure 2 shows the Nuclear Plant watermarked images after applying above said attacks. The best extracted watermarks are also shown.

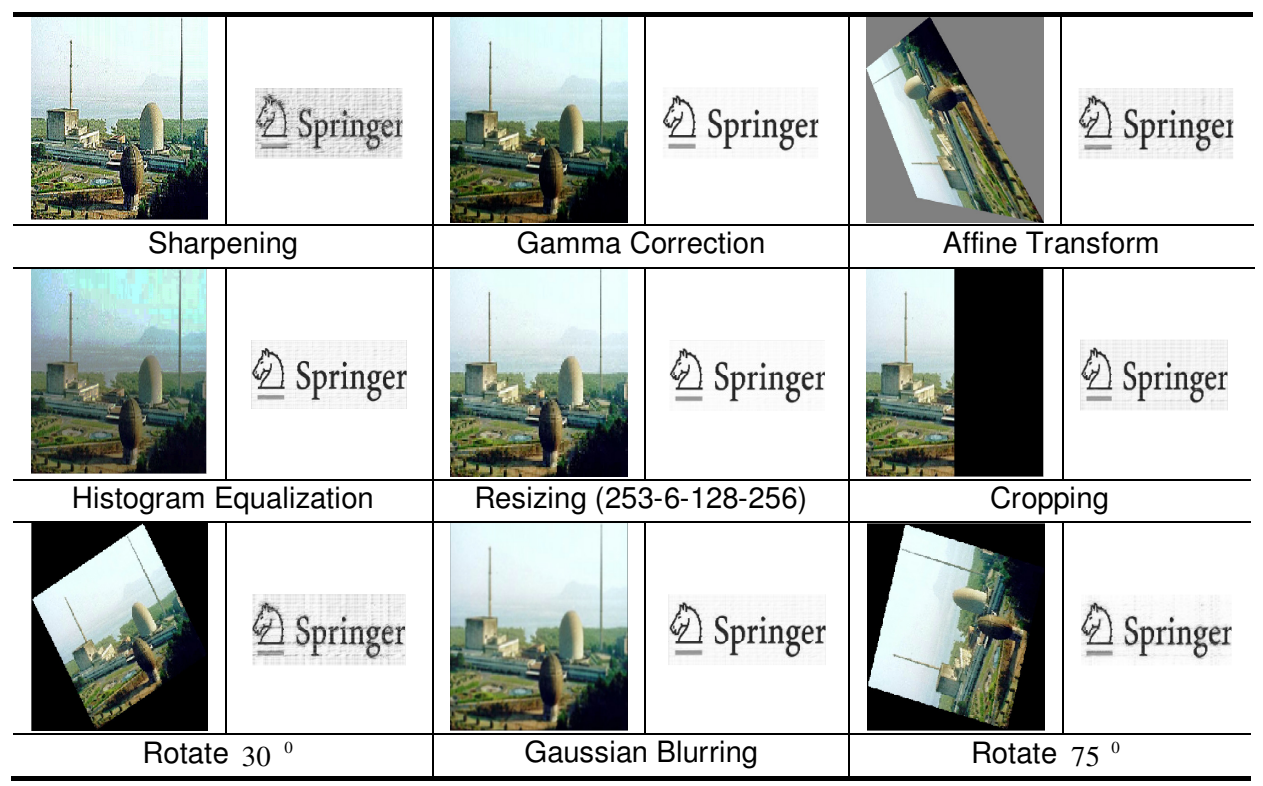

Figure 2. Extracted Springer logo from watermarked image nuclear Plant after various image Processing attacks.

The correlation coefficients of best extracted watermark image after different image processing attacks are in table 2. The results reveal that extracted watermark images have good correlation coefficients. 
The International Journal of Multimedia \& Its Applications (IJMA) Vol.3, No.1, February 2011

Table 2. Correlation coefficients of the best extracted watermark images under different image processing attacks.

\begin{tabular}{|l|l|l|l|l|l|l|l|l|}
\hline \multirow{2}{*}{ Attacks } & \multicolumn{7}{|c|}{ Watermarked Images } \\
\cline { 2 - 9 } & Lena & Barbara & Goldhill & Redfort & Boat & Taj & Status & Nuclear \\
\hline Sharpening & 0.4247 & 0.4749 & 0.6266 & 0.6414 & 0.7124 & 0.7782 & 0.7797 & 0.6066 \\
\hline $\begin{array}{l}\text { Gamma } \\
\text { Correction }\end{array}$ & 0.8522 & 0.9789 & 0.9561 & 0.9726 & 0.9908 & 0.9861 & 0.9881 & 0.9859 \\
\hline Transform & 0.6232 & 0.5902 & 0.9557 & 0.6090 & 0.9605 & 0.8347 & 0.8102 & 0.8929 \\
\hline $\begin{array}{l}\text { Histogram } \\
\text { Equalization }\end{array}$ & 0.6384 & 0.7095 & 0.9670 & 0.5856 & 0.8407 & 0.9157 & 0.7727 & 0.9501 \\
\hline Resizing & 0.9864 & 0.9897 & 0.9956 & 0.9957 & 0.9955 & 0.9961 & 0.9959 & 0.9948 \\
\hline $\begin{array}{l}\text { Cropping(left } \\
\text { half) }\end{array}$ & 0.5178 & 0.7299 & 0.9879 & 0.7478 & 0.9869 & 0.8725 & 0.7128 & 0.9307 \\
\hline $\begin{array}{l}\text { Cropping(right } \\
\text { half) }\end{array}$ & 0.7402 & 0.5003 & 0.9284 & 0.6611 & 0.9682 & 0.9681 & 0.7652 & 0.6058 \\
\hline Rotate $30^{\circ}$ & 0.4575 & 0.4134 & 0.8622 & 0.4334 & 0.9283 & 0.8247 & 0.6950 & 0.7203 \\
\hline Rotate 75 ${ }^{\circ}$ & 0.6164 & 0.5623 & 0.8964 & 0.5503 & 0.7832 & 0.8931 & 0.8527 & 0.8701 \\
\hline Gaussian Blur & 0.9232 & 0.9086 & 0.9717 & 0.9882 & 0.9826 & 0.9943 & 0.9951 & 0.9735 \\
\hline
\end{tabular}

\subsection{Experiment 3 and Results}

The next experiment was performed to see the effect of adding different types of noises such as Salt \& Peppers and Speckle Noise on watermarked images with varying density/variance within the interval [0.001, 0.1]. Amplification factor is set to 0.01 for embedding watermark. Tables 3 and 4 depict the correlation coefficients of best extracted watermark image after applying salt $\&$ peppers and speckle noise attacks respectively. The results show that correlation coefficient of extracted watermark decreases as the noise density/variance increases.

Table 3. Correlation coefficients of the best extracted watermark images under Salt \& Peppers noise

\begin{tabular}{|l|l|l|l|l|l|l|l|l|}
\hline \multirow{2}{*}{ Density } & \multicolumn{7}{|c|}{ Watermarked Images } \\
\cline { 2 - 9 } & Lena & Barbara & Goldhill & Redfort & Boat & Taj & Statue & Nuclear \\
\hline 0.001 & 0.9858 & 0.9888 & 0.9899 & 0.9887 & 0.9874 & 0.9710 & 0.9064 & 0.9890 \\
\hline 0.003 & 0.9777 & 0.9751 & 0.9545 & 0.8671 & 0.9504 & 0.9246 & 0.8692 & 0.9212 \\
\hline 0.005 & 0.8758 & 0.9112 & 0.9371 & 0.8580 & 0.9252 & 0.8894 & 0.8016 & 0.9193 \\
\hline 0.007 & 0.8647 & 0.8923 & 0.9231 & 0.7411 & 0.9073 & 0.7493 & 0.7874 & 0.8002 \\
\hline 0.009 & 0.7980 & 0.8705 & 0.8896 & 0.7133 & 0.8044 & 0.7351 & 0.6653 & 0.7412 \\
\hline 0.01 & 0.7148 & 0.8125 & 0.7888 & 0.6984 & 0.7582 & 0.7162 & 0.6615 & 0.6715 \\
\hline 0.03 & 0.4726 & 0.4882 & 0.5406 & 0.3894 & 0.4609 & 0.4137 & 0.3899 & 0.4262 \\
\hline 0.05 & 0.3533 & 0.3945 & 0.3902 & 0.3052 & 0.3678 & 0.3029 & 0.3051 & 0.3067 \\
\hline 0.07 & 0.2831 & 0.3047 & 0.3538 & 0.2508 & 0.2854 & 0.2505 & 0.2570 & 0.2685 \\
\hline 0.09 & 0.2394 & 0.2783 & 0.2962 & 0.2238 & 0.2629 & 0.2252 & 0.2294 & 0.2297 \\
\hline 0.1 & 0.2161 & 0.2594 & 0.2643 & 0.2103 & 0.2452 & 0.2140 & 0.2131 & 0.2259 \\
\hline
\end{tabular}


The International Journal of Multimedia \& Its Applications (IJMA) Vol.3, No.1, February 2011

Table 4. Correlation coefficients of the best extracted watermark images under Speckle noise

\begin{tabular}{|l|l|l|l|l|l|l|l|l|}
\hline \multirow{2}{*}{ Variance } & \multicolumn{7}{|c|}{ Watermarked Images } \\
\cline { 2 - 9 } & Lena & Barbara & Goldhill & Redfort & Boat & Taj & Statue & Nuclear \\
\hline 0.001 & 0.9883 & 0.9931 & 0.9880 & 0.9780 & 0.9915 & 0.9920 & 0.9684 & 0.9962 \\
\hline 0.003 & 0.9595 & 0.9825 & 0.9823 & 0.9090 & 0.9837 & 0.9790 & 0.8913 & 0.9880 \\
\hline 0.005 & 0.9338 & 0.9740 & 0.9537 & 0.8423 & 0.9619 & 0.9710 & 0.8151 & 0.9802 \\
\hline 0.007 & 0.8725 & 0.9494 & 0.9201 & 0.7699 & 0.9504 & 0.9522 & 0.7588 & 0.9644 \\
\hline 0.009 & 0.8403 & 0.9353 & 0.9071 & 0.6998 & 0.9214 & 0.9389 & 0.7054 & 0.9443 \\
\hline 0.01 & 0.7693 & 0.8978 & 0.8563 & 0.6969 & 0.8891 & 0.9210 & 0.6956 & 0.9316 \\
\hline 0.03 & 0.5696 & 0.7176 & 0.6715 & 0.4107 & 0.6666 & 0.7511 & 0.4335 & 0.7639 \\
\hline 0.05 & 0.4429 & 0.5893 & 0.4936 & 0.3272 & 0.5105 & 0.6355 & 0.3357 & 0.6468 \\
\hline 0.07 & 0.3673 & 0.4869 & 0.4327 & 0.2628 & 0.4505 & 0.5248 & 0.2934 & 0.5443 \\
\hline 0.09 & 0.3195 & 0.4056 & 0.3659 & 0.2433 & 0.3939 & 0.4622 & 0.2526 & 0.4802 \\
\hline 0.1 & 0.2991 & 0.3862 & 0.3363 & 0.2188 & 0.3612 & 0.4675 & 0.2370 & 0.4524 \\
\hline
\end{tabular}

Another experiment was performed to see the effect of decomposition filter on watermarked and extracted watermark image. We use three different filters 'pkva', ' $9 / 7$ ' and ' $5 / 3$ ' for experimentation. Table 5 shows the correlation coefficients of best extracted watermark image for different types of decomposition filters. The results reveal that correlation coefficients of extracted watermark in all cover images for filter 'pkva' is better as compared to that obtained using ' $5 / 3$ ' and ' $9 / 7$ ' filters. Tables 6 and 7 demonstrate the PSNR and SSIM values of watermarked image under different filters. The result shows that the watermarked image is less distorted when we use filter 'pkva' instead of ' $9 / 7$ ' and ' $5 / 3$ ' filters.

Table 5. Effect of decomposition filter on extracted watermark images in terms of correlation coefficients.

\begin{tabular}{|l|l|l|l|l|l|l|l|l|}
\hline \multirow{2}{*}{$\begin{array}{c}\text { Filter } \\
\text { Name }\end{array}$} & \multicolumn{7}{|c|}{ Watermarked Images } \\
\cline { 2 - 10 } pkva & Lena & Barbara & Goldhill & Redfort & Boat & Taj & Status & Nuclear \\
\hline $9 / 7$ & 0.9909 & 0.9792 & 0.9936 & 0.9932 & 0.9930 & 0.9966 & 0.9962 & 0.9942 \\
\hline $5 / 3$ & 0.9715 & 0.9731 & 0.9799 & 0.9792 & 0.9792 & 0.9821 & 0.9800 & 0.9841 \\
\hline
\end{tabular}

Table 6. Effect of decomposition filter on watermarked images in terms of PSNR.

\begin{tabular}{|l|l|l|l|l|l|l|l|l|}
\hline $\begin{array}{c}\text { Filter } \\
\text { Name }\end{array}$ & \multicolumn{7}{|c|}{ Watermarked Images } \\
\cline { 2 - 9 } pkva & 43.2226 & 42.6960 & 43.1691 & 43.1228 & 43.0486 & 43.4269 & 42.1642 & 43.7958 \\
\hline $9 / 7$ & 42.7426 & 41.9539 & 42.7565 & 42.3805 & 42.3632 & 42.6532 & 41.0858 & 42.2874 \\
\hline $5 / 3$ & 42.7353 & 42.0049 & 42.5989 & 42.4686 & 42.4536 & 42.5474 & 41.2676 & 42.4350 \\
\hline
\end{tabular}


The International Journal of Multimedia \& Its Applications (IJMA) Vol.3, No.1, February 2011

Table 7. Effect of decomposition filter on watermarked images in terms of SSIM.

\begin{tabular}{|l|l|l|l|l|l|l|l|l|}
\hline Filter & \multicolumn{7}{|c|}{ Watermarked Images } \\
\cline { 2 - 10 } Name & Lena & Barbara & Goldhill & Redfort & Boat & Taj & Status & Nuclear \\
\hline pkva & 0.9845 & 0.9947 & 0.9976 & 0.9875 & 0.9915 & 0.9856 & 0.9682 & 0.9815 \\
\hline $9 / 7$ & 0.9945 & 0.9945 & 0.9978 & 0.9828 & 0.9907 & 0.9846 & 0.9652 & 0.9757 \\
\hline $5 / 3$ & 0.9863 & 0.9949 & 0.9978 & 0.9845 & 0.9914 & 0.9862 & 0.9694 & 0.9777 \\
\hline
\end{tabular}

The next experiment was performed to show the effect of level of decomposition filter used on watermark image. Figure 3 shows the effect of level of decomposition on directional filter bank (DFB). The results reveal that third-level decomposition gives good quality of extracted watermark image.

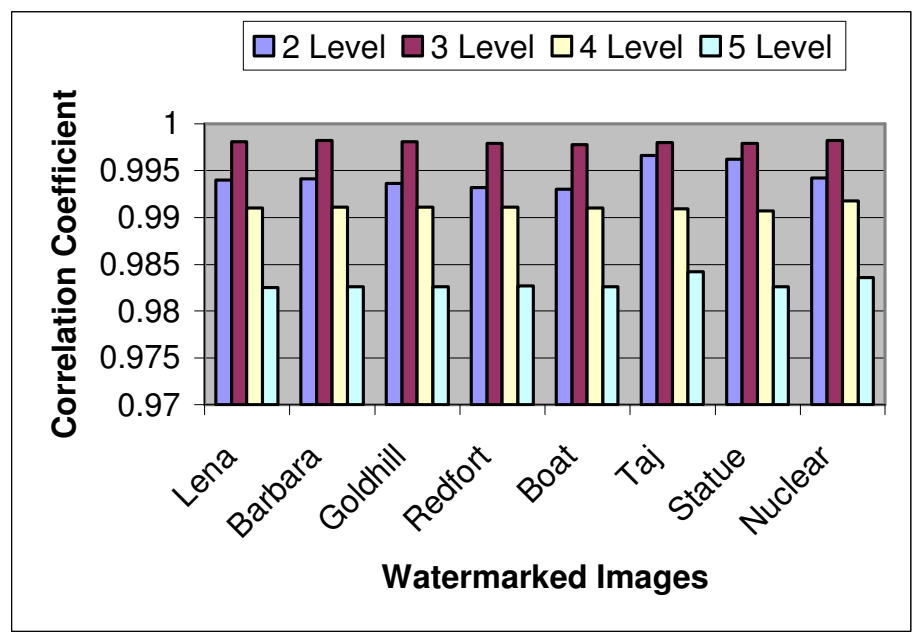

Figure 3. Effect of level of decomposition on DFB

Further simulation was done to see the effect of scaling factor on watermarked image. Tables 8 and 9 give SSIM and PSNR for different values of scaling factor on watermarked image respectively. A careful look at the tables 8 and 9 reveal that the increase in value of scaling factor results in decrease in values of both PSNR and SSIM. The best values are obtained when the scaling factor is in the range $0.03-0.01$.

Table 8. Structural Similarity metric of the watermarked images under different scaling factor

\begin{tabular}{|l|l|l|l|l|l|l|l|l|l|l|}
\hline & \multicolumn{10}{|c|}{ Scaling Factor } \\
\cline { 2 - 12 } & 0.01 & 0.03 & 0.05 & 0.07 & 0.09 & 0.1 & 0.3 & 0.5 & 0.7 & 0.9 \\
\hline Nuclear & 0.982 & 0.982 & 0.981 & 0.981 & 0.981 & 0.981 & 0.968 & 0.952 & 0.933 & 0.915 \\
\hline Taj & 0.985 & 0.985 & 0.985 & 0.985 & 0.984 & 0.984 & 0.972 & 0.952 & 0.930 & 0.907 \\
\hline Statue & 0.968 & 0.968 & 0.967 & 0.965 & 0.963 & 0.961 & 0.924 & 0.883 & 0.844 & 0.808 \\
\hline Goldhill & 0.997 & 0.997 & 0.997 & 0.997 & 0.997 & 0.996 & 0.988 & 0.975 & 0.962 & 0.949 \\
\hline Boat & 0.991 & 0.991 & 0.991 & 0.991 & 0.990 & 0.990 & 0.978 & 0.963 & 0.947 & 0.931 \\
\hline
\end{tabular}


The International Journal of Multimedia \& Its Applications (IJMA) Vol.3, No.1, February 2011

Table 9. PSNR of the watermarked images under different scaling factor

\begin{tabular}{|l|l|l|l|l|l|l|l|l|l|l|}
\hline & \multicolumn{10}{|c|}{ Scaling Factor } \\
\cline { 2 - 12 } & 0.01 & 0.03 & 0.05 & 0.07 & 0.09 & 0.1 & 0.3 & 0.5 & 0.7 & 0.9 \\
\hline Nuclear & 43.79 & 43.79 & 43.72 & 43.56 & 43.28 & 43.11 & 38.19 & 34.26 & 31.43 & 29.28 \\
\hline Taj & 43.43 & 43.42 & 43.36 & 43.20 & 42.92 & 42.75 & 38.00 & 34.14 & 31.37 & 29.23 \\
\hline Statue & 42.16 & 42.16 & 42.10 & 41.97 & 41.68 & 41.54 & 36.97 & 33.42 & 31.01 & 29.17 \\
\hline Goldhill & 43.16 & 43.15 & 43.10 & 42.98 & 42.75 & 42.61 & 38.20 & 34.56 & 31.97 & 29.98 \\
\hline Boat & 43.04 & 43.04 & 43.03 & 43.00 & 42.75 & 42.59 & 37.85 & 34.00 & 31.21 & 29.06 \\
\hline
\end{tabular}

\section{Conclusions}

This paper has presented a colour image watermarking scheme for embedding a gray scale watermark into colour cover image using CT together with DCT and SVD. The watermarked images were subjected to common image processing attacks. The simulation results depict the efficiency of the scheme in terms of quality of watermarked image and the robustness against the attacks. The extracted watermarks also have better correlation coefficients.

\section{REFERENCES}

[1] M., Arnolad, M., Schumucker, \& A., Wolthusen, (2003) Techniques and Applications of Digital Watermarking and Content Protection, Artech House, USA.

[2] Ali Al Haj, (2007) "Combined DWT-DCT Digital Image Watermarking”, J. Computer Science, Vol. 3, pp740-746.

[3] K., Rao, \& P.,Yip, (1990) Discrete Cosine Transform: algorithms, advantages, applications, Academic Press, USA.

[4] V., Potdar, S., Han, \& E., Chang, (2005) "A Survey of Digital Image Watermarking Techniques", IEEE International Conference on Industrial Informatics, pp709-716.

[5] A., Piva, M., Barni, \& F., Bartolini, (1998) "Copyright protection of digital images by means of frequency domain watermarking”, SPIE Conference on Mathematics of Data/Image Coding, Compression and Encryption, pp.25-35.

[6] M., N., Do, \& M., Vitterli, (2005) "The Contourlet Transform: An efficient directional multiresolution image representation", IEEE Transcations on Image Processing, Vol. 14, No. 12, pp2091-2096.

[7] B., Chandra Mohan, \& S., Srinivas Kumar, (2008) "Robust Digital Watermarking Scheme using Contourlet Transform”, J. Computer Science and Network Security, Vol. 40, No. 2, pp43-51.

[8] R., H., Bamerger, \& M., J., T., B., Smith, (1992) "A filter bank for the directional decomposition of images: theory and design", IEEE Transcations on Signal Processing, Vol. 40, pp882-893.

[9] P., J., Burt, \& E., H., Adelson, (1983) “The Laplacian pyramid as a compact image codes”, IEEE Transcations on Communications, Vol. 31, pp532-540.

[10] M., Jaylakshmi, S., N., Merchant, \& U., B, Desai, (2006) "Blind Watermarking in Contourlet Domain with Improved Detection", International Conference on Intelligent Information Hiding and Multimedia Signal Processing,.

[11] M., N., Do, \& M., Vitterli, (2006) "Contourlets: a directional multiresoluational image representation”, International Conference on Image Processing, pp497-501.

[12] S., Ghannam, \& F., E., Z., Abou-Chadi, (2009) "Enhancing Performance of Image Watermarks Using Contourlet Transform”, National Radio Science Conference, pp1-9. 
The International Journal of Multimedia \& Its Applications (IJMA) Vol.3, No.1, February 2011

[13] Z., Wang, A., C., Bovik, H., R., Sheikh, \& E., P. Simoncelli, (2004) "Image Quality assessment: From error measurement to structural”, IEEE Transcations on Image Processing, Vol. 13, No. 1, pp601-612.

[14] Sanjay, Rawat, \& Balasubramanian Raman, (2010) "A New Robust Watermarking Scheme for Color Images”, IEEE International Advance Computing Conference, pp206-209.

[15] Z., Q., Hong, (1991) “Algebraic feature extraction of image for recognition", J. Pattern Recognition, Vol. 24, pp211-219.

[16] A., Shnayderman, A., Gusev \& A., Eskicioglu, (2004) “A Multidimensional Image Quality Measure Using Singular Value Decomposition", SPIE Image Quality and System Performance, pp82-92.

[17] A. Basso, F., Bergadano, D., Cavagnino, V., Pomponiu \& A., Vernone, (2009) “A Novel Blockbased Watermarking Scheme Using the SVD Transform”, J. Algorithms, Vol.2, pp46-75. 